\title{
ALongitudinal Study to Identify the Causes of Mortality in Fayoumi and Rhode Island Red Chickens and the Immune Status of the Birds against Newcastle Disease
}

\author{
Suman Das Gupta ${ }^{1}$, Paritosh Kumar Biswas ${ }^{1 *}$, M Nural Anwar², Bhobesh Chandra Dey and Nitish Chandra \\ Debnath $^{1}$ \\ ${ }^{1}$ Department of Microbiology, Chittagong Veterinary \& Animal Sciences University, Khulshii, Chittagong 4202, Bangladesh, ${ }^{2}$ Department of \\ Microbiology, University of Chittagong, Chittagong 4331, Bangladesh, ${ }^{3}$ Pahartali Zonal Poultry Farm (PZPF), Pahartali, Chittagong 4202, \\ Bangladesh
}

[Received 31 March 2007; Accepted 21 April 2007]

With a prospective longitudinal study undertaken between November 2005 and July 2006 survival rates and disease status of Fayoumi and Rhode Island Red (RIR) chickens, reared at a government poultry farm, Pahartali Zonal Poultry Farm (PZPF), were assessed. Immune status of these chickens against Newcastle disease (ND) was also determined by estimating the haemagglutination inhibition (HI) titre to Newcastle disease virus (NDV) in blood sera of the chickens. The results showed that, at the end of the 37 week, the survival rate of the Fayoumi and RIR chickens were 87 and $81 \%$ respectively. The difference of the survival rates between the two groups was significant $(p<0.05)$. Among the diseases, coccidiosis was the predominant disease affecting both the populations. The incidence rate of coccidiosis, coccidiosis plus enteritis, salmonellosis, colibacillosis and heat stroke in Fayoumi and RIR chickens was 0.095 and $0.135,0.064$ and $0.057,0.007$ and $0.010,0.006$ and 0.012 , and 0.017 and 0.016 respectively. The HI titre dynamics showed that, in case of Fayoumi (except $1^{\text {st }}$ month), HI titre was always $\geq \log _{2}{ }^{6.4}$, which was well above the protective threshold. Like Fayoumi, the highest level of $\mathrm{HI}$ titre $\left(\geq \log _{2}{ }^{6.9}\right)$ to NDV in the RIR chickens was recorded in the same month and almost identical ranges of $\mathrm{HI}$ titres to NDV were recorded in both Fayoumi and RIR chickens throughout the study period.

Keywords: Fayoumi chickens, Rhode Island Red (RIR) chickens, Newcastle disease (ND), Newcastle disease virus (NDV), Survival rate, Incidence rate, Haemagglutination inhibition (HI)

\section{Introduction}

From 1980s, small-holders' poultry farming has taken a new dimension for creating income and employment for the rural poor, vulnerable women and the unemployed youth at the village level of Bangladesh through the financial assistance of the Danish International Development Agency (DANIDA), the Asian Development Bank (ADB) and other international donor agencies $^{1}$. Over the time, in some rural areas, Bangladesh has developed a village-poultry-production chain by interlinking with at least 6 kinds of interdependent beneficiaries in a cooperative fashion ${ }^{1}$. These beneficiaries include key rearers, chick rearers, model breeders, feed sellers, egg collectors and poultry health workers ${ }^{1}$. With the technical and financial supports from the Department of Livestock Services (DLS) and different nongovernmental organizations (NGOs), it is believed that at least two millions of these beneficiaries have been enrolled in this village-poultry-production chain ${ }^{2}$.

As a vital input Rhode Island Red (RIR), Fayoumi and Sonali (a cross-bred from Fayoumi and RIR, i.e., $\mathrm{F}_{1}$ generation) chicks/ chickens, produced from the government poultry farms, are supplied to these beneficiaries ${ }^{3}$. These exotic and crossbred chickens might introduce some new disease problems to our indigenous backyard chickens. Of all diseases, Newcastle disease (ND) is the major constraint for the production of village chickens all over the developing world including Bangladesh ${ }^{4-5}$.

If not properly cared for, ND could also be transmitted from the government farm to the village level. Therefore, it is also important to evaluate the effectiveness of the vaccination regimen practiced against ND for the chickens reared in government poultry farms. Longitudinally, this study aims to observe the important diseases affecting the chickens in a government farm along with the immune status of these birds against ND.

\section{Materials and Methods}

Place, period and nature of the study

It was a prospective longitudinal study. The study was undertaken between November 2005 and July 2006. The Fayoumi and Rhode Island Red (RIR) chickens, which are the parent breeds of Sonali, were observed during this period at a government poultry farm,

*Corresponding author:

Dr. Paritosh Kumar Biswas, Professor \& Head, Department of Microbiology, Chittagong Veterinary \& Animal Sciences University, Chittagong 4202, Bangladesh Tel (Office): (031) 659093/110; Fax: 880 (031) 659492; E-mail: biswaspk2000@yahoo.com 
Pahartali Zonal Poultry Farm (PZPF), Chittagong. All laboratory works related to this study were undertaken at the Department of Microbiology, Chittagong Veterinary and Animal Sciences University (CVASU), Chittagong, Bangladesh.

\section{Study populations and their management}

One flock of each of Fayoumi and RIR with the flock size 2,750 and 1,250 birds respectively were started observing from the date of their placement at the age of day 1 into two well-separated poultry sheds. This sheds had walls (made up with bricks and cement) and bottom decimetres made of concrete; windows were covered with movable curtains. The birds were supplied with balanced ration prepared by the own feed mill of the farm. Three types of ration were formulated and supplied to the observed birds: starter, grower and layer according to their ages. The major ingredients used for a ration were maize, rice polish, soybean meal, protein concentrate and oyster shell.

\section{Vaccination schedule for the study population}

Table 1 shows the vaccination schedule undertaken by the PZPF management for birds of the both observed groups. Vaccines were given against 4 diseases - Newcastle disease (ND), infectious bursal disease (IBD), fowl pox and fowl cholera. Both live and killed vaccines were given against ND and IBD. For ND, the first vaccine administered was baby chick Ranikhet disease vaccine (BCRDV), which is prepared from the Livestock Research Institute (LRI), Mohakhali, Dhaka using the F strain of Newcastle disease virus (NDV), a lentogenic strain of NDV. As booster dose the same vaccine was administered at day 21 . To boost the immunity further killed ND vaccine was given at day 12 and 120; while a stronger live vaccine called Ranikhet disease vaccine (RDV), made from Mukteswar strain (a mesogenic one) of NDV, was given twice at day 60 and 150.

Table 1. Vaccination schedule undertaken for the observed Fayoumi and RIR birds at PZPF

\begin{tabular}{ll}
\hline Vaccine given at age (Day ) & Name of vaccine given \\
\hline 1 & IBD/Gumboro (Vi Bursa CE ${ }^{\mathrm{R}}$ ) \\
5 & BCRDV \\
12 & ND-killed (Imopest ${ }^{\mathrm{R}}$ ) \\
17 & Gumboro (Booster) \\
21 & BCRDV (Booster) \\
32 & Fowl pox \\
60 & RDV \\
75 & Fowl cholera \\
120 & ND-killed \\
150 & RDV \\
\hline
\end{tabular}

RIR = Rhode Island Red chicken; PZPF = Pahartali Zonal Poultry Farm, Chittagong; IBD = Infectious bursal disease; BCRDV = Baby chick Ranikhet disease vaccine; $\mathrm{ND}$ = Newcastle disease; $\mathrm{RDV}=$ Ranikhet disease vaccine

Determination of survival rates of Fayoumi and RIR chickens Occurrences of diseases were considered the numbers of failures and taking this into account (the total number of failures), the survival rate of Fayoumi and RIR chickens were calculated separately by using the total number of birds survived at a definite period as numerator divided by the initial number of birds placed for the observation as the denominator. The survival progression was shown in Kaplan-Meier survival curve.

\section{Sample collection}

The two flocks were visited everyday and organ sample - liver, heart, bone marrow and gastro-intestinal tract were collected from the dead chickens for laboratory investigation. These organ samples were collected separately in polythene-bags and kept frozen at the department of microbiology at $-84^{\circ} \mathrm{C}$ until investigated. Blood samples were collected aseptically from the chickens. After collection of blood, the samples were left for 2-3 $\mathrm{h}$ in order to separate the serum from the blood clots. When separated from blood clots a serum sample was transferred into a sterile Eppendorf tube (3.0-ml capacity) and then preserved chemically with merthiolate at a concentrate of 1:10,000 to prevent bacterial contamination. The serum samples were also kept frozen at $-84^{\circ} \mathrm{C}$ until analyzed.

Diagnoses of diseases and measure applied for the load of a disease

Diagnoses of diseases were made by clinical signs, post-mortem examination findings supported with laboratory investigations according to OIE manual ${ }^{6}$. Incidence rate (IR) was applied to estimate the load of a disease in a study population. For a disease either in the Fayoumi or RIR chicken population, IR was calculated by using the total number of cases recorded during the observation as numerator divided by the total bird-months at risk observed as the denominator.

\section{Assessment of HI titre to ND virus}

To assess the level of haemagglutination inhibition (HI) titre to NDV in vaccinated chickens, HI test was performed in V-bottomed micro-well plastic plates. The test procedure was conducted as per the methodology described in the OIE manual ${ }^{6}$. For preparation of red blood cells (RBCs) used in the test, blood was taken from chickens that have not been primed with Newcastle disease (ND) vaccine. This collected blood was pooled in an equal volume of Alsever's solution. After that, the blood was washed thrice in PBS before used as a 1\% RBCs (packed cell volume) suspension. Serum was tested in two fold dilutions. For virus suspension, 4 HA strength ' $F$ ' strain of NDV was used. For positive control, pooled serum collected from five-ND vaccinated chickens was used, and for negative control, uninfected allantoic fluid collected from 9-day's embryonated chicken eggs was used.

\section{Data analyses}

All data were entered the spread-sheet program (Excel; Microsoft Company) and then transferred to computerized statistical package stata-7 for data management and summary. The differences in the level of HI titres for the two chicken breeds at different intervals were calculated using ANOVA while the difference of the survival rates between Fayoumi and RIR chickens was shown using a $\chi^{2}$ test.

\section{Results}

Survivability of chickens of the study populations

Figure 1 portrays (Kaplan-Meier graph) the survival rates of Fayoumi and Rhode Island Red (RIR) chickens on day basis from 
day 1 up to day 257. Altogether 322,850 and 196,068 bird-day at risk for Fayoumi and RIR chickens respectively were observed. And 357 Fayoumi and 237 RIR chickens died during the observation. About 99\% Fayoumi chicks survived by the end of three weeks of age and within the next 3 weeks the survival rate declined by only $1 \%$. However, higher death rates of Fayoumi chickens were recorded in the next 4 weeks started from the beginning of $7^{\text {th }}$ week and ended in the $10^{\text {th }}$, resulting sharp declining in the survival rate from 99 to $88 \%$. A very low weekly mortality of Fayoumi chickens was recorded in the rest period of the observation, commencing from the $11^{\text {th }}$ week until the observation was over at end of the week 37. At the end of the last week, the survival rate of the Fayoumi chickens was about $87 \%$. Seven RIR chicks died in the first week, rendering the weekly survival rate of RIR chicks to be $99 \%$ (at the end of the first week). A slow but steady weekly mortality (1-2\%) of RIR chickens was observed from the first week and up to $5^{\text {th }}$ week of their age, by the end of $5^{\text {th }}$ week the survival rate of RIR chicks was $96 \%$. Compared with Fayoumi a higher mortality of RIR chickens was observed from the $6^{\text {th }}$ week and this higher rate of mortality continued until the $11^{\text {th }}$ week commenced. By the end of $10^{\text {th }}$ week the survival rate of RIR chickens reduced to $83 \%$, which is significantly lower $(p<0.5)$ than the Fayoumi chickens (compared at this same age limit). After this age, a very low mortality of RIR chickens occurred and by the end of the observation the survival rate declined only by $2 \%$, resulting the overall survival rate of RIR chickens about $81 \%$.

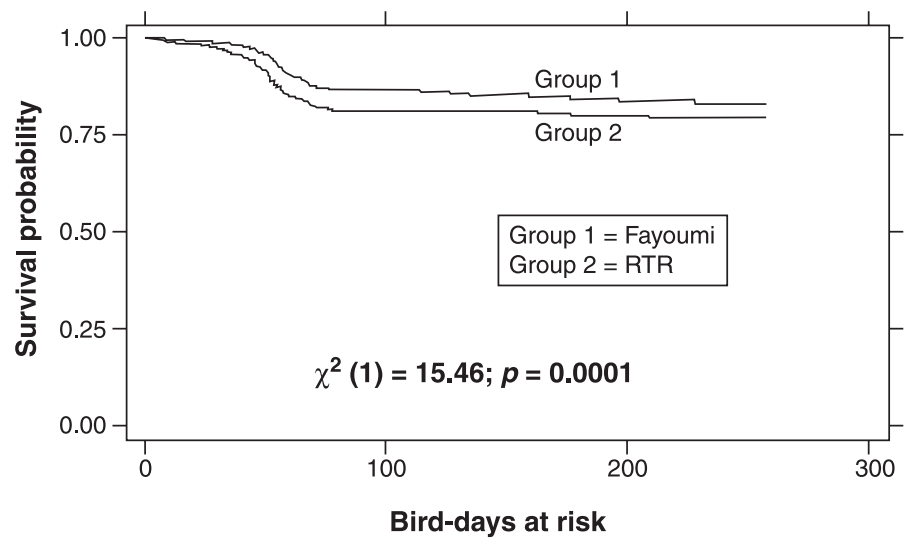

Figure 1. Survival rates of Fayoumi and RIR chickens reared at Pahartali Zonal Poultry Farm (PZPF) on bird-days at risk basis. Survival rates are shown by Kaplan-Meier graph from 10 November 2005 to 24 October 2006. RIR = Rhode Island Red chicken.

\section{Frequencies of different diseases}

During the observation, 357 and 237 chickens belonged to Fayoumi and RIR chickens died with the presence of 5 different disease conditions such as coccidiosis, coccidiosis plus necrotic enteritis, salmonellosis, colibacillosis (colisepticaemia) and heat stroke (Table 2). Coccidiosis was the predominant disease affecting both the populations.
Table 2. Frequencies of different diseases observed in two breeds of chickens reared in the Pahartali Zonal Poultry Farm (PZPF)

\begin{tabular}{lccc}
\hline Disease & \multicolumn{2}{c}{ Frequencies in two breeds } & $p$ value \\
\cline { 2 - 3 } & Fayoumi $^{\mathrm{a}}$ & RIR $^{\mathrm{b}}$ & \\
\hline Coccidiosis & 164 & 119 & 0.307 \\
Coccidiosis plus necrotic enteritis & 110 & 50 & 0.009 \\
Salmonellosis & 12 & 9 & 0.778 \\
Colibacillosis & 10 & 11 & 0.234 \\
Heat stroke & 30 & 14 & 0.255 \\
Undiagnosed & 31 & 34 & 0.030 \\
(decomposed samples included) & & & \\
\hline
\end{tabular}

RIR = Rhode Island Red chicken; ${ }^{\text {a,b }}=$ Respective population size $=$ No. at the beginning + No. at the end of observation $/ 2 ;^{a}=1718 ;^{b}=883$

Incidence rates of different diseases

Quantification of the five diseases, namely, coccidiosis, coccidiosis plus necrotic enteritis, salmonellosis, colibacillosis and heat stroke, based on the measure incidence rate is shown in Table 3. The incidence rates of major two disease conditions coccidiosis and coccidiosis plus necrotic enteritis in Fayoumi chickens were 0.095 and 0.064 respectively. These mean during this study period 95 and 64 Fayoumi chickens per 1,000 chickens might have died due to coccidiosis and its concomitant occurrence with necrotic enteritis, respectively. Salmonellosis was present in both the populations investigated but the incidence rate of it was very low (0.007 in case Fayoumi vs. 0.010 in case of RIR). Like salmonellosis, colibacillosis can also be found with a very lower rate; $6 / 1,000$ and 12/1,000 in Fayoumi and RIR chickens respectively. Heat stroke, a non-infectious disease recorded with a magnitude of 16-17/1,000 birds of each of the two populations.

Haemagglutination inhibition (HI) titre dynamics to Newcastle disease virus (NDV) in the study populations

In Fayoumi chickens, the lowest HI titre was recorded in the serum samples collected from the first month; except this month, the $\mathrm{HI}$ titre was always $\geq \log _{2} 6.4$ in all other monthly samples (Table 4). And the highest HI titre (almost $\log _{2}{ }^{10}$ ) to NDV in the Fayoumi chickens was found in the samples collected for the $4^{\text {th }}$ month. Like Fayoumi, the highest level of HI titre to NDV in the RIR chickens was recorded in the same month. The lowest $\mathrm{HI}$ titre in this population was observed when the birds attained the age of 8-month. The HI titre dynamics showed that all of the chickens in the both populations had a higher level of antibodies, which were well above the protective threshold. Also, in temporal serum samples, almost identical ranges of HI titres to NDV were recorded in both Fayoumi and RIR chickens observed through this prospective longitudinal study $(p>0.05)$. 
Table 3. Incidence of major diseases in the two study populations of Fayoumi and RIR chickens reared in the Pahartali Zonal Poultry Farm (PZPF)

\begin{tabular}{llll}
\hline Disease & IR-Fayoumi $(\mathrm{n}=1,718)$ & IR-RIR $(\mathrm{n}=883)$ & IRR \\
\hline Coccidiosis & $0.095(0.109,0.081)$ & $0.135(0.158,0.112)$ & 0.704 \\
Coccidiosis plus necrotic enteritis & $0.064(0.076,0.052)$ & $0.057(0.072,0.042)$ & 1.123 \\
Salmonellosis & $0.007(0.011,0.003)$ & $0.010(0.017,0.003)$ & 0.700 \\
Colibacillosis & $0.006(0.009,0.002)$ & $0.012(0.019,0.005)$ & 0.500 \\
Heat stroke & $0.017(0.023,0.011)$ & $0.016(0.024,0.008)$ & 1.063 \\
\hline Total & 0.189 & 0.230 & 0.822 \\
\hline
\end{tabular}

$\mathrm{IR}$ = Incidence rate; IRR = Incidence rate ratio; RIR = Rhode Island Rhode chicken

Table 4. Dynamics of haemagglutination inhibition (HI) titre to Newcastle disease virus (NDV) in chickens of the two study populations

\begin{tabular}{lccccc}
\hline Day/Month & \multicolumn{4}{c}{$\log _{2 \mathrm{HI}}$ titre $(\mathrm{C} \pm \mathrm{SEM})^{*}$} & \multirow{2}{*}{$p$ value } \\
\cline { 2 - 5 } & No. & Fayoumi & No. & RIR & \\
\hline $7^{\text {th }}$ day & 5 & $2^{7.4} \pm 0.9$ & 0 & - & - \\
$1^{\text {st }}$ month & 5 & $2^{5.2} \pm 2.3$ & 0 & - & - \\
$2^{\text {nd }}$ month & 18 & $2^{7.9} \pm 1.0$ & 21 & $2^{8.1} \pm 0.9$ & 0.62 \\
$3^{\text {rd }}$ month & 15 & $2^{7.9} \pm 2.5$ & 15 & $2^{7.9} \pm 1.3$ & 1.0 \\
$4^{\text {th }}$ month & 20 & $2^{9.8} \pm 2.7$ & 22 & $2^{9.9} \pm 1.1$ & 0.79 \\
$6^{\text {th }}$ month & 28 & $2^{8.4} \pm 0.6$ & 23 & $2^{8.1} \pm 1.3$ & 0.46 \\
$8^{\text {th }}$ month & 10 & $2^{6.4} \pm 1.1$ & 11 & $2^{6.9} \pm 0.9$ & 0.29 \\
Total & 101 & $2^{8.1} \pm 0.2$ & 92 & $2^{8.4} \pm 0.2$ & 0.247 \\
\hline
\end{tabular}

${ }^{*} \mathrm{HI}$ titre at the level of $\log _{2} 5$ was considered as the protective threshold. RIR = Rhode Island Rhode chicken

\section{Discussion}

About 87\% Fayoumi and 81\% Rhode Island Red (RIR) chickens could survive up to the 37 week of age. This was probably the first time in Bangladesh that in a Department of Livestock Services (DLS)-regulated farm, the survival rates of both Fayoumi and RIR chickens were studied. To calculate the survival rates of Fayoumi and RIR chickens all deaths were counted and the loss of chickens attributable to the other reasons (except deaths) was excluded.

Coccidiosis caused by Eimeria tenella was the predominant disease of chickens belonged to both the Fayoumi and RIR populations. The incidence of coccidiosis in the Pahartali Zonal Poultry Farm (PZPF), Chittagong was higher than the incidence recorded in chickens in the Mirpur Central Poultry Farm, Dhaka ${ }^{7}$ and in chickens raised under intensive/commercial system in Bangladesh $^{8}$. Chickens of all ages and breeds are susceptible to Eimeria tenella infection ${ }^{9}$. Newly hatched chicks are sometimes not fully susceptible to infection because of insufficient chymotrypsin and bile salts in the intestine to cause excystation ${ }^{9}$. The oocysts of coccidia build up along the growth of the chicks and the number of the oocysts then decline when the birds become immune to further infection ${ }^{10}$ because it is a self-limiting disease ${ }^{9}$. In our study, we recorded the clinical onset of coccidiosis in the RIR and Fayoumi chicks at the age of 6 and 7 weeks respectively, however, the peak of the clinically overt disease was recorded at the age of 9 weeks - such a long course of overt coccidiosis was rarely reported.

Coccidiosis might also cause immune-suppression that help opportunistic or other obligate pathogens to cause lesions in the damaged or undamaged intestinal mucosa. A substantial number of dead chickens, both Fayoumi and RIR, had both coccidiosis and necrotic enteritis. It is speculated that immuno-suppression resulted from coccidiosis (of Eimeria tenella origin) could be the predisposing factor to develop necrotic enteritis ${ }^{11}$.

Salmonellosis is a vertically transmitted disease. It can be found both in endemic and epidemic forms affecting poultry birds in Bangladesh. Although a very low incidence of salmonellosis was recorded, nevertheless, it delineates that salmonellosis is present in PZPF. The low incidence of salmonellosis in this study differs from that of the findings of $\mathrm{Huq}^{7}$ who reported high incidence of the disease in Fayoumi chickens reared in the Mirpur Central Poultry Farm. Also this result differs from Talha ${ }^{8}$ who found a higher proportional mortality caused by salmonellosis in chickens raised in commercial system. Identical but low incidence of salmonellosis was recorded in each of the two studied breeds of chickens, Fayoumi and RIR. There is a report on the presence of salmonellosis in Fayoumi birds reared in a government farm as mentioned above ${ }^{7}$ but the incidence of salmonellosis in RIR chickens (reared in any DLS-regulated farms) might have not been reported before this study.

In this study, the magnitude of colibacillosis in any of the two chicken populations studied in the PZPF was lower than the incidence of it in the Fayoumi chickens based in the Mirpur Central Poultry Farm, Dhaka ${ }^{7}$. And in this central poultry farm, most of the colibacillosis cases occurred in the young birds, is however in agreement with the present study. Also the incidence of colibacillosis in the present study was lower than that of reported in the broody hen chicks of the Smallholder Livestock Development Project-2 (SLDP-2) area ${ }^{12}$ and that of chickens reared under commercial system ${ }^{8}$.

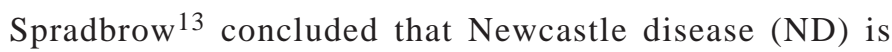
insuperable from the chickens (particularly backyard types) of the developing countries. To contain it proper vaccines and vaccinations should be chosen. Based on the findings of this 
study it is recommended that the current vaccination regimens practiced by the PZPF-management should be kept untouched; although a killed vaccine is expensive but it generates a high level of antibodies. At least one vertically transmitted disease, salmonellosis, is present in the PZPF. It's presence in other DLSregulated farms was also acknowledged ${ }^{14}$. Chickens of PZPF can be monitored serologically in the view to establishing vertically disease free parent flocks to supply disease free chickens to the rural areas of Bangladesh. Coccidiostat should be incorporated in the poultry feed of this farm to reduce the incidence of coccidiosis. Hygienic litter management should also be emphasized.

\section{Acknowledgements}

The study was a part of an MPhil programme and the research project was financed by the DANIDA-SLDP-2 Research Fellowship Programme. With the deepest sense of gratitude, we would like to thank to the concerned authorities for providing financial supports to carry out the study.

\section{References}

1. Ahamed N. 2002. Components of Bangladesh semi-scavenging poultry model. In People Fight Poverty with Poultry: Learning from the Bangladesh Experience. Workshop Report, 20-24 ${ }^{\text {th }}$ October 2002. Dhaka, Bangladesh.

2. Karim MJ. 2003. Current pattern in poultry with special emphasis on parasites and their methods on control. In Proceedings of $3^{\text {rd }}$ International Poultry Show and Seminar, pp 119-123. Bangladesh China Friendship Conference Centre (BCFCC), Sher-e-Bangla Nagar, Dhaka, Bangladesh, 28 $8^{\text {th }}$ February-2 ${ }^{\text {nd }}$ March 2003. Dhaka, Bangladesh.

3. Fattah KA. 2000. Poverty alleviation poultry development strategies in Bangladesh. In Poultry as a Tool in Poverty Eradication and Promotion of Gender Equality (Dolberg F \& Petersen PH eds), pp 151-164. Proceedings of the First Annual Scientific Conference, $22^{\text {nd }}$ $26^{\text {th }}$ March 1999, Tune Lanboskole, Denmark.
4. Aini I. 1990. Indigenous chicken production in South-east Asia. World Poultry Sci J. 46: 51-57.

5. Chowdhury SI, Chowdhury TIMFR, Sarker AJ, Amin MM \& Hossain WIMA.1982. Studies on Newcastle disease in Bangladesh. The role of residual maternal antibody on immune response and selection of an optimum age for primary vaccination of chicks. Research Report, pp 12-22. Dhaka, Bangladesh.

6. OIE. 2002. Manual of standards for diagnostic tests and vaccines. Office International Des Epizooties, Paris. Available at: www.oie.int. Assessed 12 January 2006.

7. Huq MR. 2002. Longitudinal study of the causes of mortality of chickens in parent stock flocks of the Department of Livestock Services (DLS) of Bangladesh with a special emphasis on Escherichia coli infection. MSc Thesis, p 66. The Royal Veterinary and Agricultural University, Dyrlaegevej, Frederiksbeurg, Denmark.

8. Talha AFSM, Hossain MM, Chowdhury EH, Bari ASM, Islam MR \& Das PM. 2001. Poultry diseases occurring in Mymensingh district of Bangladesh. Bangladesh Veterinarian. 18(2): 20-23.

9. McDougald LR \& Reid WM. 1997. Coccidiosis. In Diseases of Poultry, $10^{\text {th }}$ edn, pp 865-883. Mosby-Wolfe, London.

10. Reyna PS, Mathis GF \& McDougald LR. 1982. A survey of sensitizing anticoccidial from broiler chickens in Brazil and Argentina. Avian Dis. 31: 287-292.

11. Al-sheikhly F \& Al-Saieg A. 1980. Role of coccidia in the occurrence of necrotic enteritis of chickens. Avian Dis. 24: 324-333.

12. Biswas PK. 2004. A longitudinal study to identify the causes on the mortality of 'Sonali' birds and broody hen chicks of key beneficiaries in the SLDP-2 area. Smallholder Livestock Development Project-2 (SLDP-2) Applied Research Project. Final Report, p 78. Chittagong, Bangladesh.

13. Spradbrow PB.1993. Newcastle disease in village chickens. Poultry Sci Rev. 5: 57-96.

14. Bell JG. 2004. Disease control strategies in the smallholder livestock development project in five southern districts of Bangladesh. Proceedings of a Final Research Coordination Meeting, FAO/IAEA Division of Nuclear Techniques in Food and Agriculture, pp 38-41. Vienna, Austria, $24^{\text {th }}-28^{\text {th }}$ May 2004. Vienna, Austria. 